\title{
Re-irradiation of silver nanoparticles obtained by laser ablation in water and assessment of their antibacterial effect
}

M. Fernández-Arias ${ }^{a *}$, M. Boutinguiza ${ }^{\mathrm{a}}$, J. del Val ${ }^{\mathrm{a}}$, E. Medina ${ }^{\mathrm{b}}$, D. Rodríguez ${ }^{\mathrm{b}}$, A. Riveiro $^{a}$, R. Comesaña ${ }^{c}$, F. Lusquiños ${ }^{\text {a }}$, F.J. Gil ${ }^{d}$, J. Pou ${ }^{a}$

${ }^{a}$ Applied Physics Dpt., University of Vigo, EEI, Lagoas-Marcosende, Vigo, E-36310, Spain

${ }^{\mathrm{b}}$ Biomaterials, Biomechanics and Tissue Engineering Group, Materials Science and Metallurgical Engineering Dept., UPC-Barcelona TECH, Sant Adrià del Besòs, Barcelona, E-08930, Spain

' Materials Eng., Applied Mech., and Construction Dpt., University of Vigo, LagoasMarcosende, E-36310, Vigo, Spain

d School of Dentistry. Universitat Internacional de Catalunya, Barcelona, Spain

*Corresponding author at: Applied Physics Dpt., University of Vigo, EEI, LagoasMarcosende, Vigo, E-36310, Spain. Tel.: +34986812216; fax: +34986812201.

E-mail address: monfernandez@uvigo.es (M. Fernández-Arias) 


\begin{abstract}
In this work silver nanoparticles were produced by using two nanosecond $\mathrm{Nd}: \mathrm{YVO}_{4}$ lasers operating at 1064 and $532 \mathrm{~nm}$ respectively to ablate a silver target submerged in pure deionized water. Part of the resulting colloidal solution was injected as a fine stream by a compressed air system and re-irradiated one and three times with the same laser to resize and get uniform nanoparticles.

The obtained nanoparticles by ablation and re-irradiation consisted of crystalline Ag nanoparticles with a bimodal size distribution. The particle size has been reduced by subsequent laser re-irradiation with both laser sources, reaching a $40 \%$ of mean size reduction. Inhibitory effects on the proliferation of Staphylococcus aureus was demonstrated on silver nanoparticles obtained after re-irradiation with the infrared laser.
\end{abstract}

Keywords: Silver nanoparticles, laser ablation, resizing, antibacterial effects 


\section{Introduction}

It is estimated that by 2050,10 million persons will die each year due to the resistance of bacteria to antibiotics [1]. This is a huge problem that even today is causing 700.000 fatalities per year. Use of nanoparticles (NPs) could be an alternative to today's antibiotics, since NPs are able to locally destroy bacteria, but preserving the integrity of the surrounding tissues [2].

During the last years, silver nanoparticles have been widely studied because of their antibacterial properties [3-9]. In this way, different studies demonstrated the influence of size and shape of NPs in terms of bactericidal efficacy [7, 10-12]. Therefore, synthesizing nanoparticles with the adequate size distribution, morphology and crystallinity is very important. There are various methods based on physical, chemical or biologic processes to produce silver nanoparticles [13-17]. Obviously each technique has its own pros and cons . Regarding the use as bactericidal agent in human beings, is important to note that most of these methods imply the use of precursors, solvents or involve chemical reactions which can result in the contamination of the obtained nanoparticles, that could be harmful not only to bacteria but also to the surrounding tissues.

Laser ablation of solids in liquids (LASL) allows controlling the size and shape by tuning the processing parameters, but also leading to obtain pure nanoparticles with no need of any additional reagent [18]. In the last decade several groups used this technique for the production of silver NPs [19-24]. Modifications of the original technique such as using a high speed rotating target [25] or a wire target in a liquid jet [26] led to improvements on size of the nanoparticles and on the productivity respectively. With the objective of avoiding any type of contamination, laser ablation of a silver plate was carried out also in open air, obtaining NPs with rounded shape and narrow size distribution [27-28]. 
In the present work, we report the synthesis of silver nanoparticles by LASL in water using two different laser sources and the subsequent fragmentation of the obtained solutions following a somehow similar strategy as that of Wagener and Barcikowski for producing organic NPs from powders instead of a solid target [29]. Results and formation process, including influence of wavelength are discussed. Antibacterial activity is also studied with Staphylococcus aureus (a gram-positive aerobic bacteria) for being one of the major multidrug-resistant pathogens [9,30], which typically causes skin infections or even pneumonia, endocarditis and osteomyelitis [31].

\section{Materials and methods}

\subsection{Laser Ablation}

Silver foils with $99.99 \%$ of purity were cleaned and sonicated to be used as laser ablation target. As shown in Figure 1, the target was fixed inside a glass vessel filled with deionized water up to $1 \mathrm{~mm}$ over the upper surface of the silver foil. Two different laser sources working at different wavelengths were used in the process (see Table 1). The first system was a diode-pumped $\mathrm{Nd}: \mathrm{YVO}_{4}$ laser providing pulses of $14 \mathrm{~ns}$ at wavelength of $532 \mathrm{~nm}$ with $0.26 \mathrm{~mJ}$ of pulse energy. The second laser source was a $\mathrm{Nd}: \mathrm{YVO}_{4}$ laser providing pulses of $20 \mathrm{~ns}$ at $1064 \mathrm{~nm}$ of wavelength and $0.33 \mathrm{~mJ}$ of pulse energy.

In all experiments, the laser beam was focused on the upper surface of the target and was kept in relative movement with respect to the metallic plate at $50 \mathrm{~mm} / \mathrm{s}$ of scanning speed. In both cases the processing time for the LASL was 5 minutes, taking place a change in the color of the solution, becoming yellowish/brown in the first minutes of the process. 


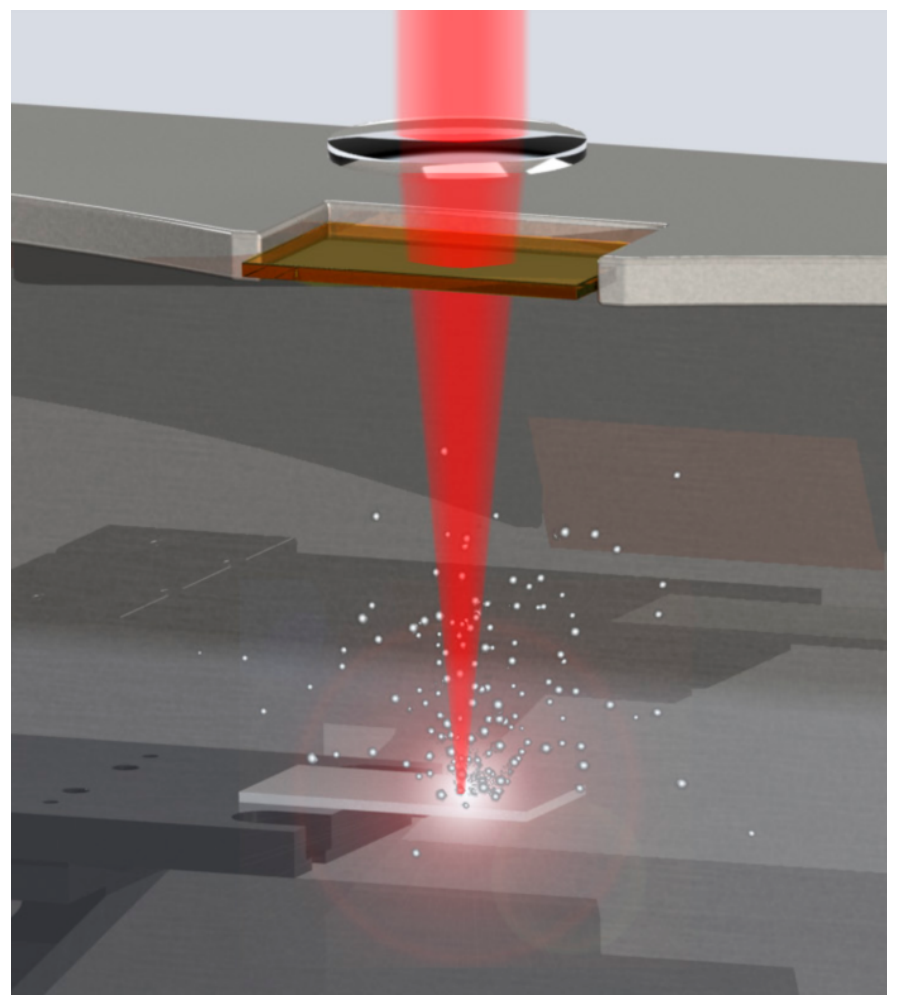

Fig. 1. Laser ablation process

Table 1. Processing parameters

\begin{tabular}{|c|c|c|c|c|c|c|}
\hline \multicolumn{2}{|l|}{ Laser source } & $\begin{array}{c}\text { Wavelength } \\
\text { (nm) }\end{array}$ & $\begin{array}{c}\text { Pulse } \\
\text { length } \\
\text { (ns) }\end{array}$ & $\begin{array}{c}\text { Frequency } \\
\text { (kHz) }\end{array}$ & $\begin{array}{c}\text { Average } \\
\text { power } \\
\text { (W) }\end{array}$ & $\begin{array}{c}\text { Scanning } \\
\text { speed } \\
\text { (mm/s) }\end{array}$ \\
\hline $\begin{array}{c}\text { Nanosecond } \\
\text { laser }\end{array}$ & Green & 532 & 14 & 20 & 12 & 50 \\
\cline { 2 - 7 } & IR & 1064 & 20 & 20 & 20 & 50 \\
\hline
\end{tabular}

\subsection{Re-Irradiation}

Part of the obtained solutions from the LASL have been re-irradiated once and three times using the same laser sources used before. Table 2 shows the different samples produced and analyzed in this study. To achieve the fracture of the largest number of nanoparticles in suspension, a system that uses compressed air to get the normal incidence of the laser beam on a reduced and continuous flow of solution has been used. The air pressure used 
during the process was 0.5 bar. This pressure was enough to keep a continuous jet and to ensure that laser beam strikes on a homogeneous area at the outlet of the capillary.

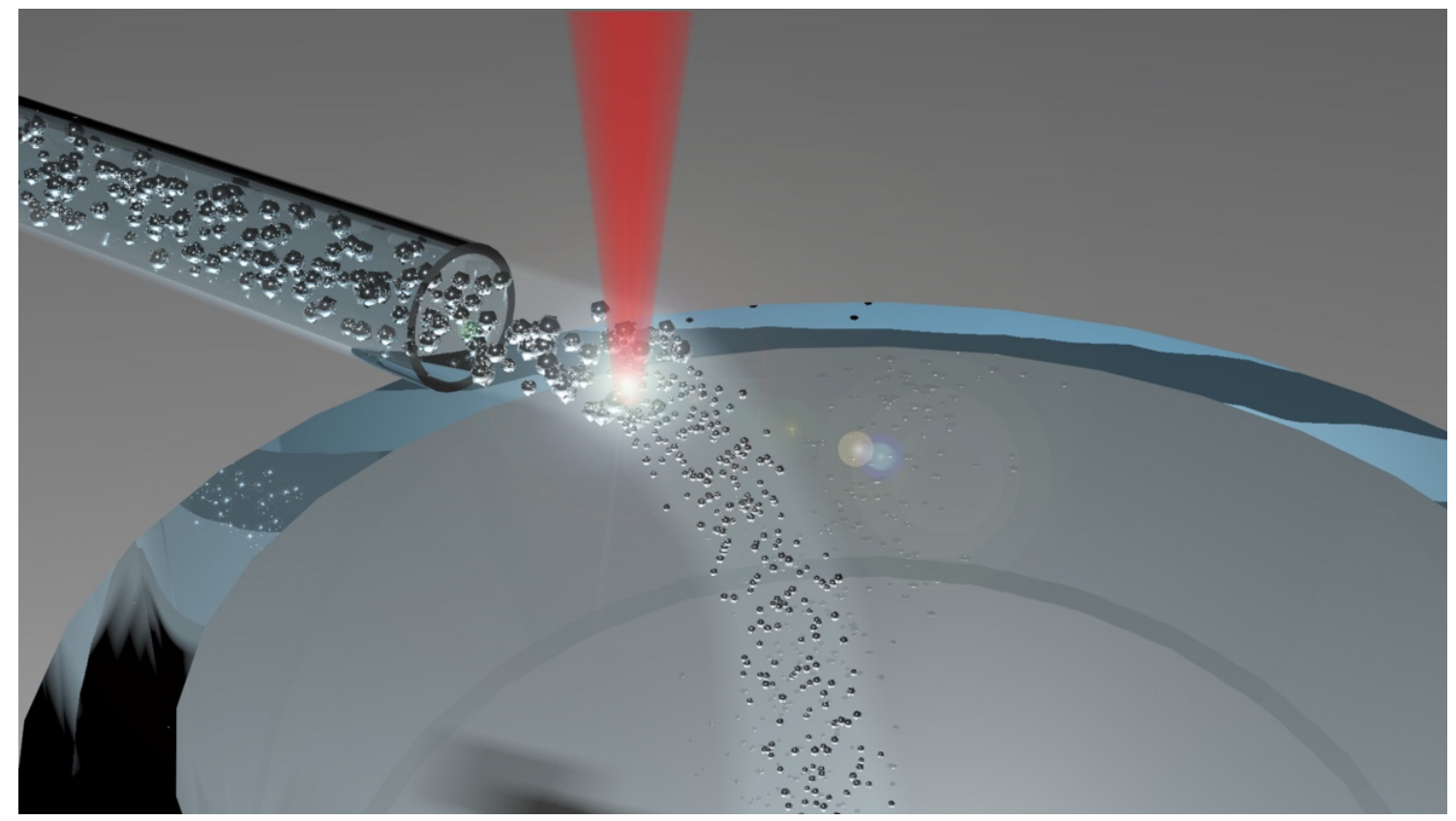

Fig. 2. Pressurized air system scheme

Table 2. Set of samples produced and analyzed

\begin{tabular}{|l|l|l|}
\hline Sample & Laser source & Treatment \\
\hline a.1 & Green & As produced \\
\hline a.2 & Green & 1 time re-irradiation \\
\hline a.3 & Green & 3 times re-irradiation \\
\hline b.1 & IR & As produced \\
\hline b.2 & IR & 1 time re-irradiation \\
\hline b.3 & IR & 3 times re-irradiation \\
\hline
\end{tabular}

\subsection{Sample preparation and characterization technics}

After each experiment, samples of the obtained colloidal suspensions were dropped on carbon-coated copper micro-grids and allowed to dry for characterization. In order to analyze the particle size, morphology and crystalline structure of the obtained nanoparticles, High-Resolution Transmission Electron Microscopy (HRTEM) images 
were obtained using a JEOL-JEM 2010F FEG transmission electron microscope equipped with a slow digital camera scan, using an accelerating voltage of $200 \mathrm{kV}$ and provided with an energy dispersive X-Ray Spectrometer (EDS) to reveal the qualitative elemental composition. X-ray diffraction (XRD) analysis was carried out in order to corroborate the crystal structure by means of a PANanalytical X'Pert Pro X-ray diffractometer using monochromated $\mathrm{Cu}-\mathrm{K} \alpha$ radiation $(\lambda=1.54 \AA)$ over the $30-90^{\circ} 2 \theta$ range with step size of $0.02^{\circ}$. The UV-vis absorption spectrum of the colloidal suspensions was measured in the range from 190 to $800 \mathrm{~nm}$ using a Hewlett Packard HP 8452 spectrophotometer. The stability of the colloidal suspensions was studied by means of the Z-Potential measurements, carried out with a Zetasizer Nano ZS ZEN3600 from Malvern Instruments.

To assess the bactericidal activity of the obtained NPs, the MIC (minimum inhibitory concentration) evaluation was performed with a gram-positive bacteria, Staphylococcus aureus subsp. aureus CECT 435 (Colección Española de Cultivos Tipo) using different dilutions (from 1:2 to 1:100) of each colloidal solution with a concentration of $300 \mathrm{mg} / \mathrm{L}$. The different silver NPs colloids were added (in triplicate) to the wells of a sterile 96well plate already containing the bacterial suspension in culture media (BHI). To ensure homogeneity, the obtained solutions were previously submerged in an ultrasonic bath for 25 minutes at room temperature. During the assay the plate was incubated at $37^{\circ} \mathrm{C}$ and the absorbance at $600 \mathrm{~nm}$ was measured every $15 \mathrm{~min}$ for 13 hours using a multimode microplate reader (Infinite $200 \mathrm{PRO}$, Tecan). 


\section{Results and discussion}

\subsection{Size, morphology, composition and crystallography}

Figures 3 and 4, show TEM images of Ag nanoparticles obtained with green and IR lasers and their corresponding size distribution. The represented histograms were obtained by measuring the diameter of about 300 particles of each sample.
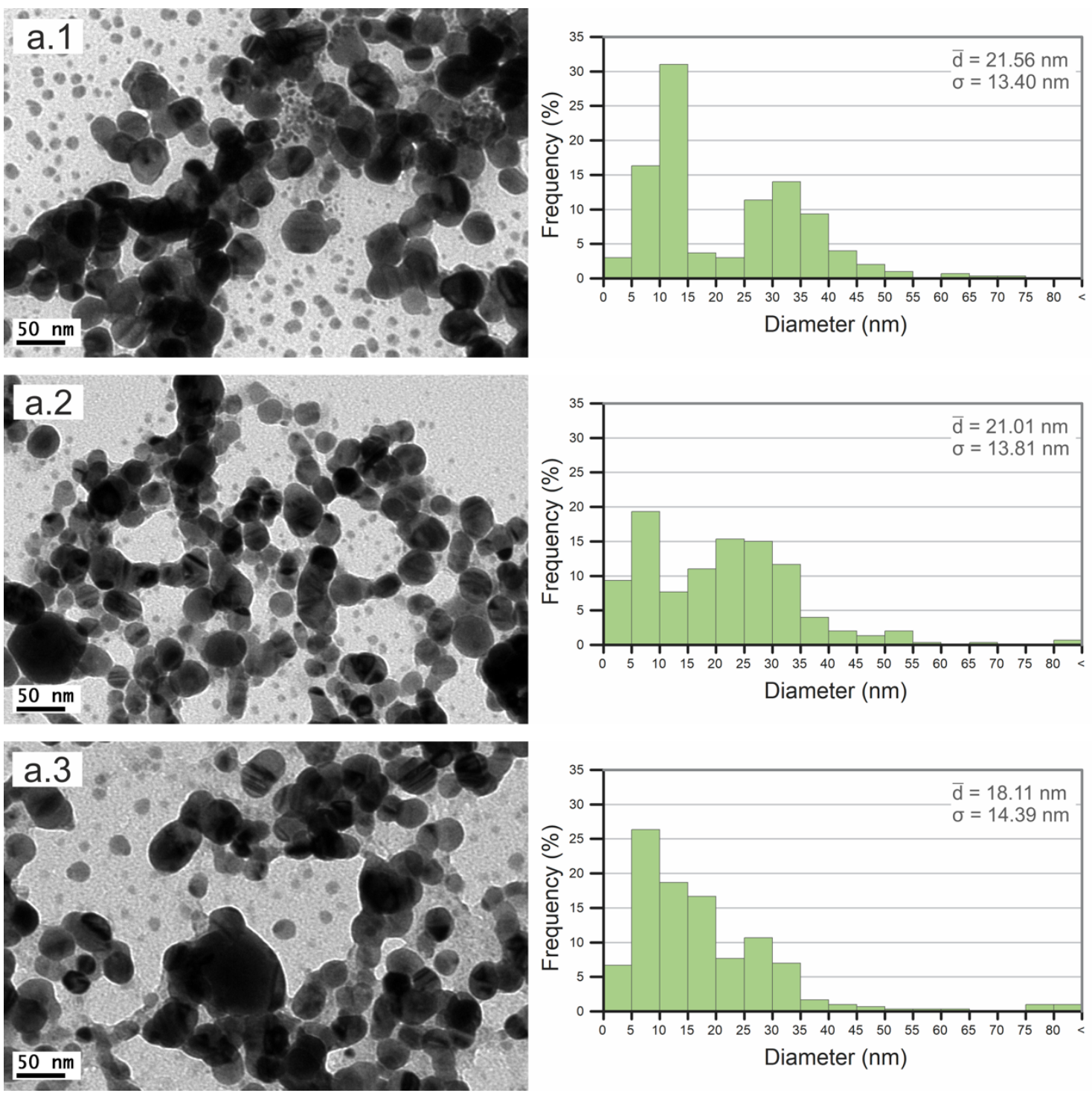

Fig. 3. TEM micrographs and size distribution of Ag nanoparticles obtained in water using a $\mathrm{Nd} \mathrm{YVO}_{4}$ laser operating at $532 \mathrm{~nm}$. a.1) by laser ablation of $\mathrm{Ag}$ foil, a.2) by one re-irradiation, a.3) after 3 reirradiations. 

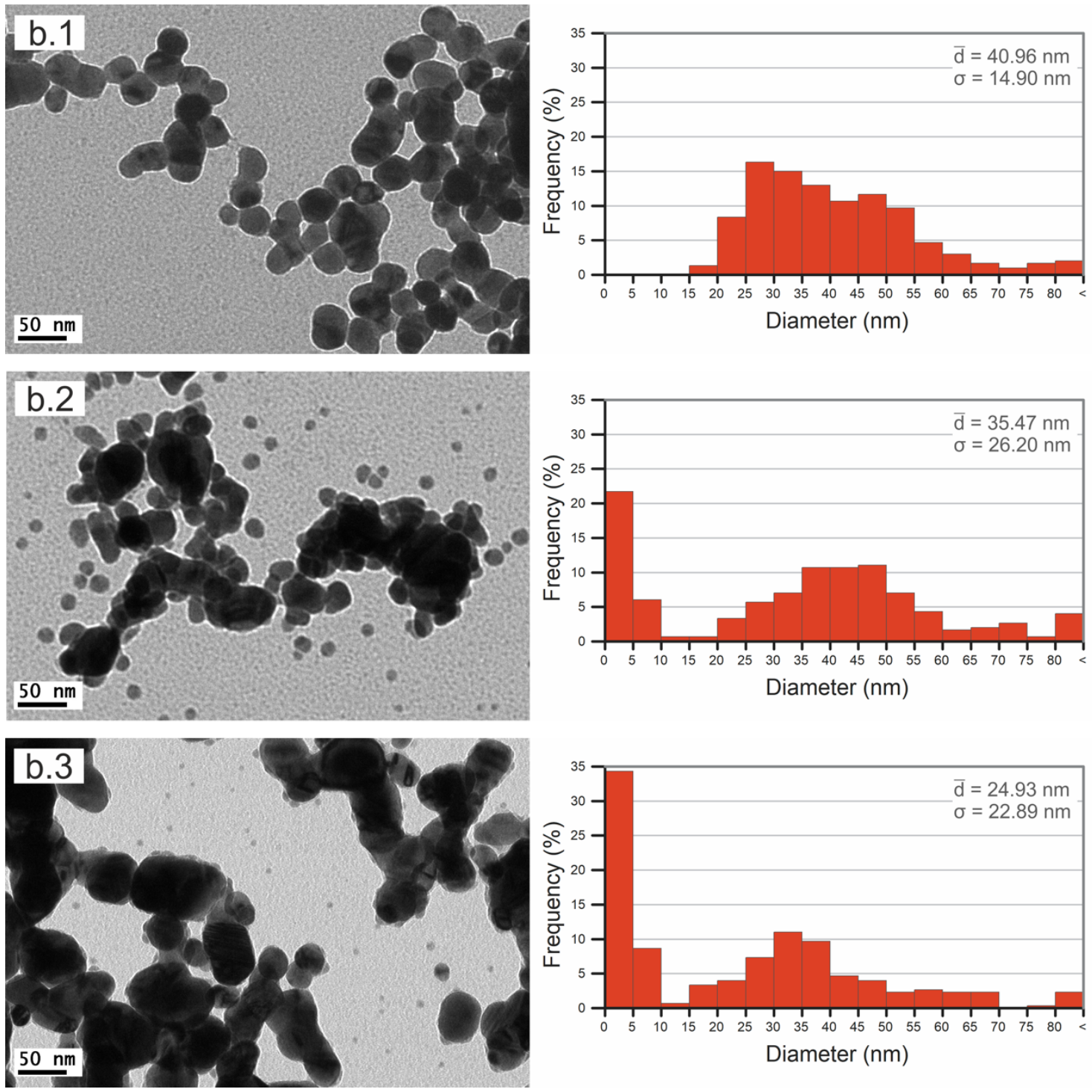

Fig.4. TEM micrographs and size distribution of $\mathrm{Ag}$ nanoparticles obtained in water using a $\mathrm{Nd} \mathrm{YVO}_{4}$ laser operating at $1064 \mathrm{~nm}$. b.1) by laser ablation of Ag foil, b.2) by one re-irradiation, b.3) after 3 re-irradiations.

Note that the silver nanoparticles obtained by LASL with both green and IR lasers, exhibit rounded shape with certain tendency to agglomeration. This is due to the formation mechanism of nanoparticles and the thermal regime taking place between laser beam and the target. When the laser beam strikes on the Ag foil, the incident radiation is absorbed heating up the silver above its melting point. Because of the high power delivered in the few nanoseconds lasting the laser pulse, the energy density surpasses the ablation threshold of the material and lead to the plasma formation. In water, the plasma plume is confined and it cools down more rapidly than in air [32] forming spherical nanoparticles. 
Subsequently, the nanoparticles size was reduced by laser re-irradiation. This process known as photo thermal ablation, causes a large number of small particles as well as a wider range of sizes [33] as can be seen in figures 3 and 4 .

On account of the metallic nature of the starting material, since although the great majority of the particles are fractured by the laser beam turning out in smaller ones, concurrently the absorbed radiation increases the temperature of the already formed NPs above the melting point, which melt and join with others by coalescence leading to the formation of larger nanoparticles or chains-like nanostructures.

Note that all samples show a bimodal size distribution. In addition, the number of nanoparticles with a diameter lower than $10 \mathrm{~nm}$ increased with the re-irradiation times, primarily when the process is carried out with the IR laser.

The EDS performed on each obtained sample confirms that the as-ablated as well as the re-irradiated nanoparticles are pure Ag. Moreover, all the particles obtained, even the smallest ones are crystalline. This aspect can be observed in figure 5, showing HRTEM of single nanoparticles with clear lattice fringes and their corresponding Fast Fourier Transform (FFT) as insets.

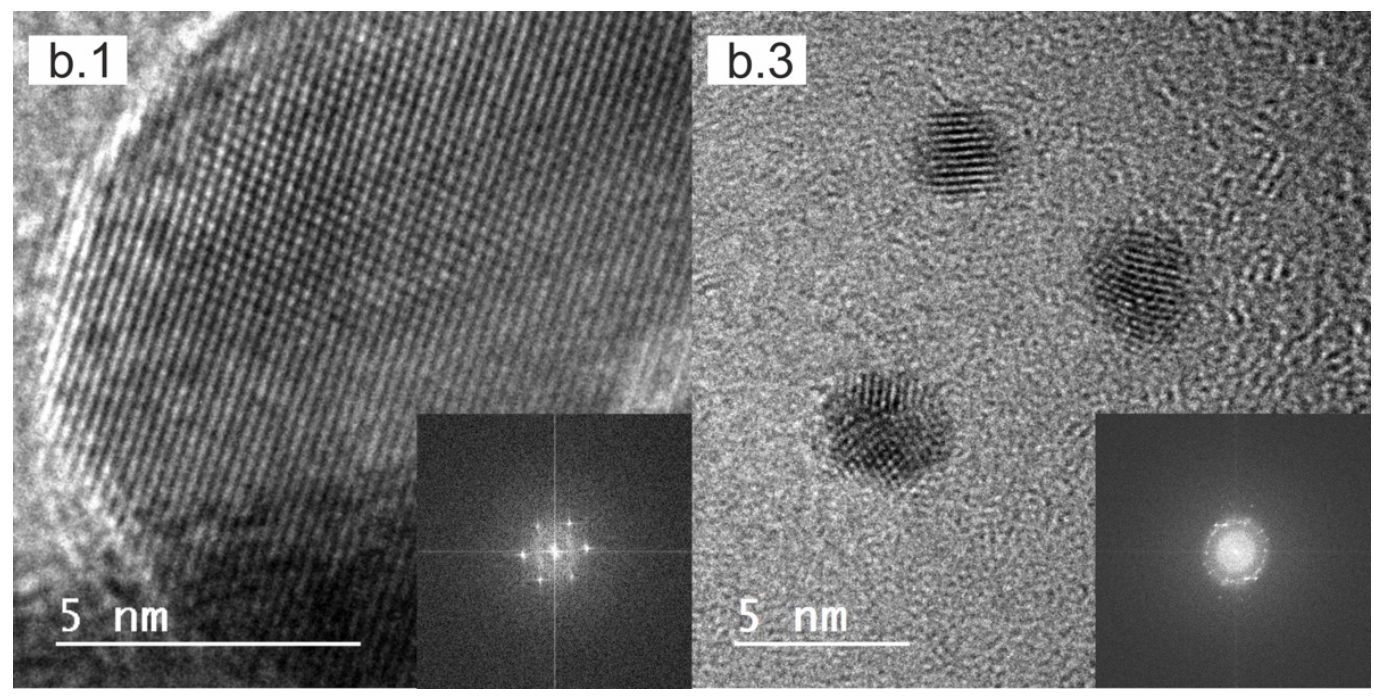

Fig.5. HRTEM images and their corresponding FFT of Ag crystalline nanoparticles obtained in water using a Nd: $\mathrm{YVO}_{4}$ laser operating at $1064 \mathrm{~nm}$. b.1) by laser ablation of $\mathrm{Ag}$ foil and b.3) after 3 re-irradiations. 
To elucidate the crystalline phases of the obtained Ag nanoparticles, the measured interplanar distances from the FFT were compared with those of metallic Ag in Table 3. The measured interplanar distances correspond with the family planes of cubic Ag (JCPDS-ICDD ref.00-004-0783). Note that the composition in terms of oxidation is not modified in the process.

Table 3. Lattice spacing measured from the FFT of Ag nanoparticles obtained by a.1), b.1) laser ablation, a.2), b.2) by one re-irradiation, a.3), b.3) by 3 re-irradiations, using $532 \mathrm{~nm}$ and $1064 \mathrm{~nm}$ respectively and compared to those of metallic Ag.

\begin{tabular}{|c|c|c|c|c|c|c|}
\hline a.1 & a.2 & a.3 & b.1 & b.2 & b.3 & Ag (hkl) \\
\hline 0.236 & 0.234 & 0.235 & 0.235 & 0.239 & 0.233 & $0.2359\left(\begin{array}{lll}1 & 1 & 1\end{array}\right)$ \\
\hline 0.209 & 0.208 & 0.214 & 0.192 & 0.222 & 0.201 & $0.2044\left(\begin{array}{lll}2 & 0 & 0\end{array}\right)$ \\
\hline- & - & - & 0.155 & - & 0.137 & $0.1445\left(\begin{array}{lll}2 & 2 & 0\end{array}\right)$ \\
\hline- & 0.116 & - & - & - & - & $0.11796\left(\begin{array}{lll}2 & 2 & 2\end{array}\right)$ \\
\hline
\end{tabular}

In order to corroborate the composition of the obtained nanoparticles, XRD were performed on the obtained nanoparticles and the precursor silver plate. The corresponding comparative diffraction patterns of the samples are depicted in figure 7. As can be seen from Fig. 7 the crystalline phase of the obtained nanoparticles is not altered by the reirradiation process, changing only size and shape.

As shown in Fig. 8, the UV-VIS spectra of the synthesized nanoparticles exhibit a peak at approximately $400 \mathrm{~nm}$, which is characteristic of surface plasmon resonance (SPR) feature of Ag nanoparticle colloidal solutions [34]. The SPR is a well-known metallic nanoparticles effect, since it is absent in individual atoms and in metallic bulk. It is associated to the nanoparticles shape, size and surrounding medium in a way that the presence of a single surface plasmon peak, implies that they are spherical $[34,35]$. The pronounced peak corresponds to the as-ablated nanoparticles while the broadening in the 
re-irradiated ones is characteristic of a wide size distribution [36] which is in agreement with the TEM observations.

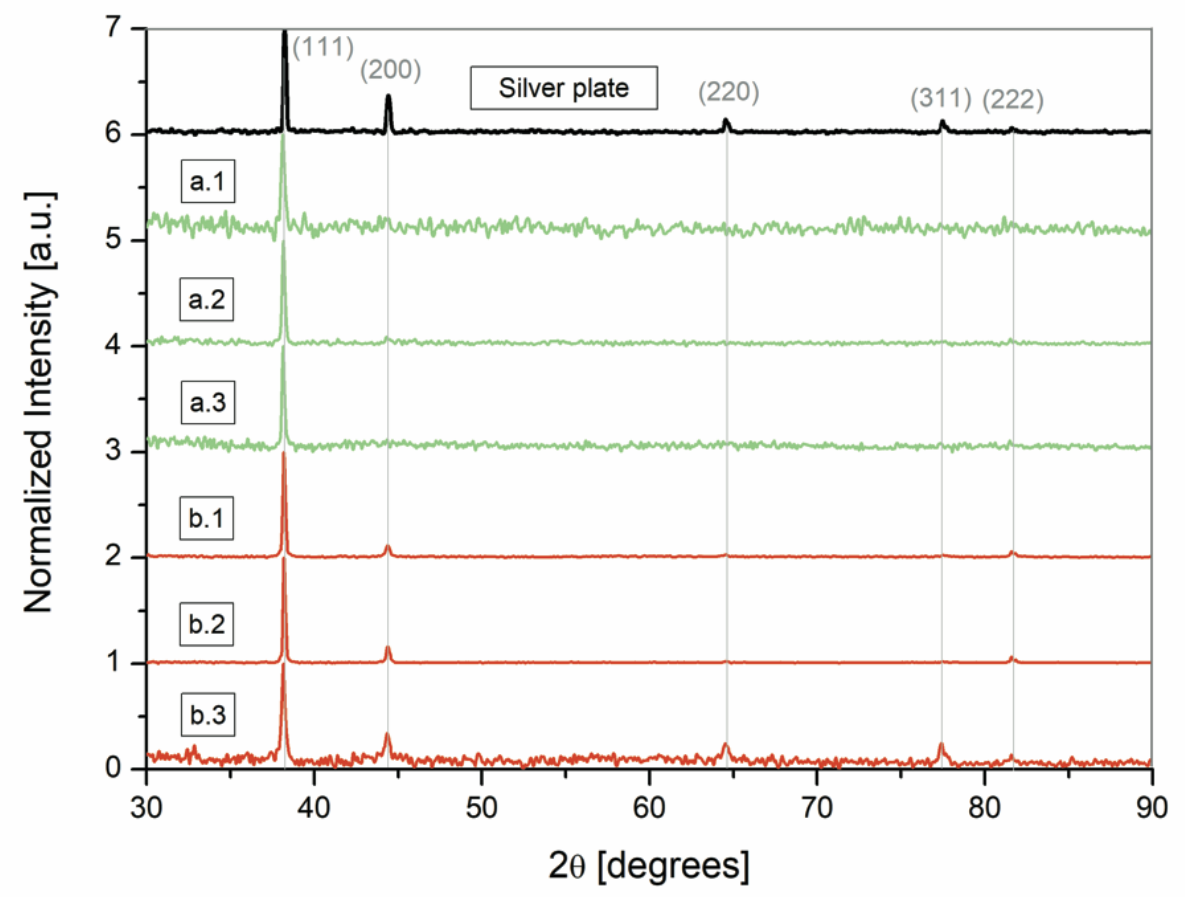

Fig.6. XRD of Ag nanoparticles obtained with a wavelength of $532 \mathrm{~nm}$ by: (a.1) LASL, (a.2) re-irradiation 1 time, (a.3) re-irradiation 3 times and with $1064 \mathrm{~nm}$ of wavelength by: (b.1) LASL, (b.2) re-irradiation 1 time, (b.3) re-irradiation 3 times.

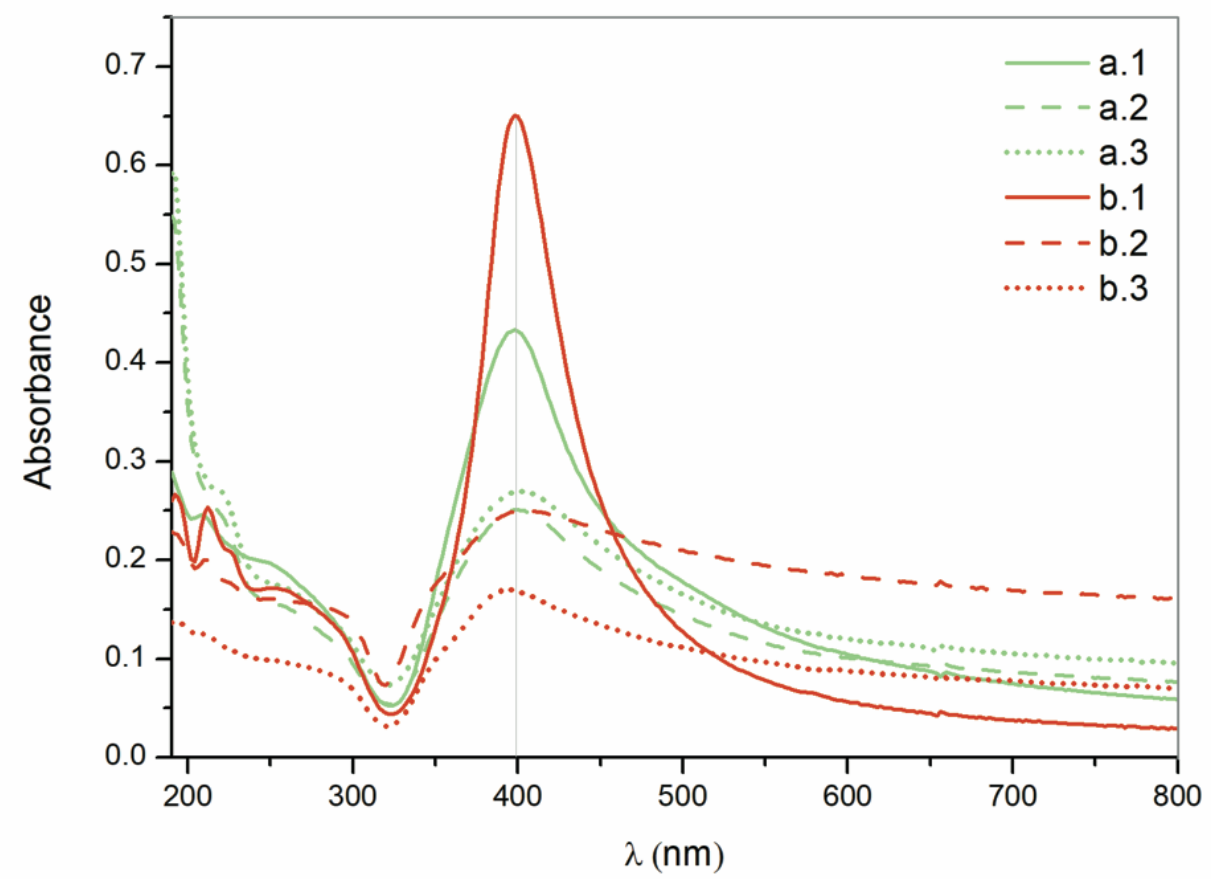

Fig.7. UV-vis spectrum of Ag nanoparticles obtained with a wavelength of $532 \mathrm{~nm}$ by: (a.1) LASL, (a.2) re-irradiation 1 time, (a.3) re-irradiation 3 times and with $1064 \mathrm{~nm}$ of wavelength by: (b.1) LASL, (b.2) reirradiation 1 time, (b.3) re-irradiation 3 times. 


\subsection{Colloid stability}

It is known that colloid stability depends on the interactions between its particles in suspension which in turn are the result of electrostatic repulsive forces and Van der Waals attractive forces. The electrokinetic potential, commonly known as Zeta potential (ZP) provides information about the interactions that take place through the measurement of the particles speed under the influence of an electric field applied.

As shown in Table 4, the measurements show a higher (absolute value) potential for the particles obtained by laser ablation than the re-irradiated particles, decreasing with the number of times the sample is re-processed. All values are negative, which reveals a basic $\mathrm{pH}$ [37] of a great importance in biological applications.

The ZP measurements were repeated after 14 days (Table 5) showing similar values.

Table 4. Z-Potential measurements

\begin{tabular}{|c|c|c|c|c|c|c|}
\hline Sample & a.1 & a.2 & a.3 & b.1 & b.2 & b.3 \\
\hline Z Potential (mV) & -23.5 & -9.18 & -1.90 & -19.03 & -19.33 & -11.5 \\
\hline
\end{tabular}

Table 5. Z-Potential measurements 14 days later

\begin{tabular}{|c|c|c|c|c|c|c|}
\hline Sample & a.1' & a.2' & a.3' & b. $1^{\prime}$ & b.2' & b.3 \\
\hline Z Potential $(\mathbf{m V})$ & -21.73 & -5.40 & -1.75 & -15.77 & -15.03 & -11.5 \\
\hline
\end{tabular}

The low values (below $10 \mathrm{mV}$ ) of the re-irradiated particles, show more instability than the as-ablated with values around $20 \mathrm{mV}$. On the other hand, there is a slight tendency to reduce the $\mathrm{Z}$ potential values, approaching to the isoelectric point (point of zero charge) which corresponds to the change in the tonality of the solution and with the flocculation of the colloids [37]. 


\subsection{Analysis of antimicrobial activity}

Antimicrobial activity was evaluated by measuring the absorbance of the different solutions at $600 \mathrm{~nm}$. Due to the different absorbance at $600 \mathrm{~nm}$ of each solution before being added to the culture medium, the relative values (to the initial absorbance value) were calculated for comparing the results. Only the 1:2 dilution showed antibacterial effects. The obtained relative absorbance of each sample is shown in Fig. 9.

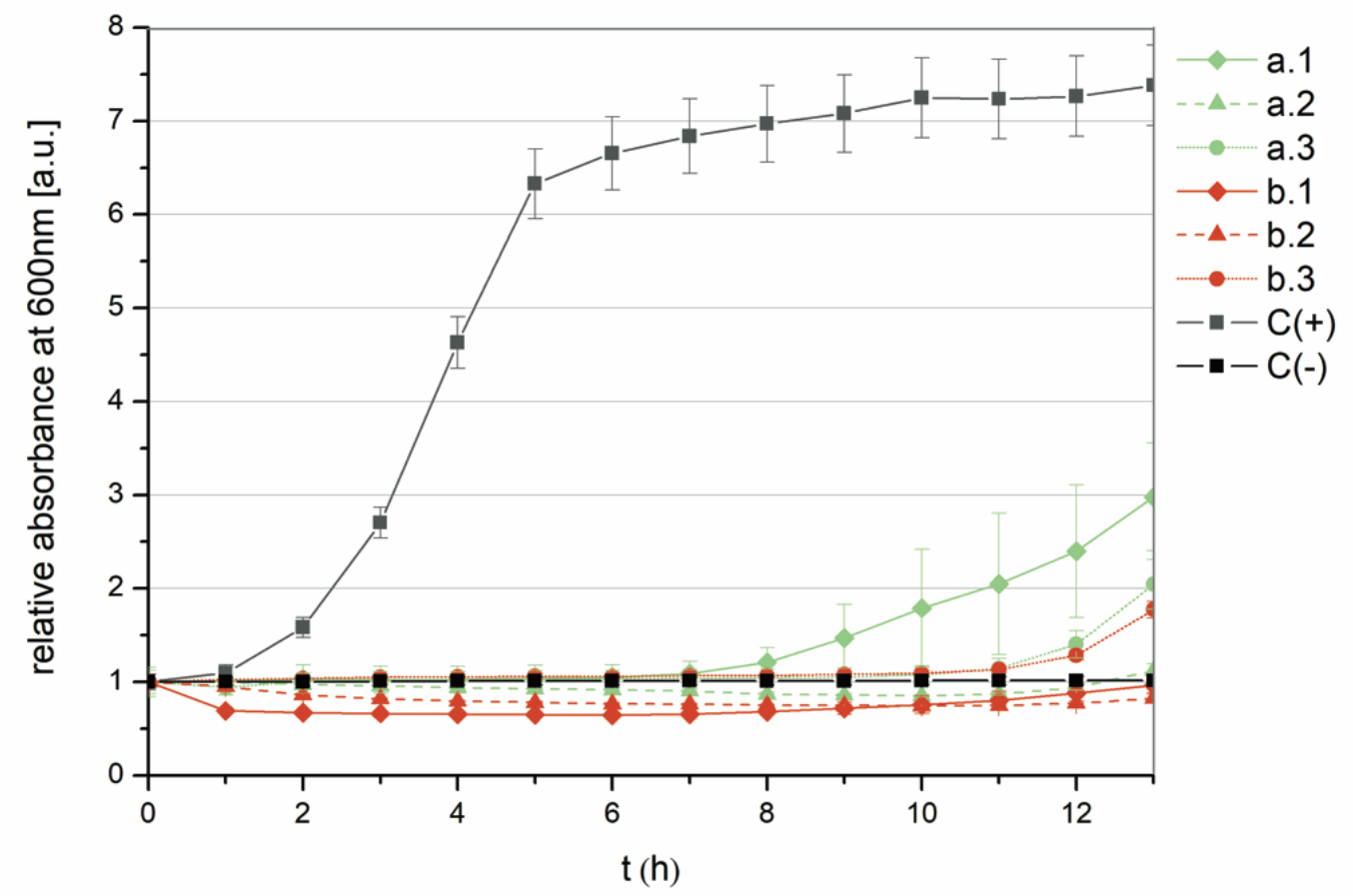

Fig.8. Growth curves of S. aureus with dilution of $1: 2$ during $13 \mathrm{~h}$ of incubation at $37^{\circ} \mathrm{C}$ in contact with the colloids. Bacterial suspension in the absence of NPs were used as control.

Taking into account that the higher the absorbance, the more bacterial growth, values were compared with a positive control (medium with bacteria but without NPs). After $13 \mathrm{~h}$ the bacterial growth values are noteworthy reduced in all samples. Silver nanoparticles obtained by re-irradiation once, exhibit a greater bactericidal capacity than their precursors (as ablated), being samples b.1 and b.2 those that show the most remarkable inhibitory effects. The rest of curves show a delay in the growth, after which the bacterial proliferation starts. 
Earlier studies $[5,11,38]$ have demonstrated that Ag NPs with a size between 1 and $10 \mathrm{~nm}$ are the ones with the greatest bactericidal effects. In this study, the smallest nanoparticles which correspond with sample a. 3 do not show the best inhibitory effects because of the formation of agglomerates, resulting in a decrease of Ag+ ions release [39], the main responsible for bacterial inhibition $[4,40]$.

Note that the samples with the highest bactericidal effect (b.2, b.1 y a.2), correspond to those that underwent a greater variation in their ZP values after 14 days.

This results are consistent with works previously reported and demonstrate not only the influence of size, but also morphology and stability of colloidal Ag NPs in the bactericidal effects $[10,41]$. In this sense, the addition of salts to the solution before irradiation, would prevent the formation of agglomerates and improve the stability of the NPs in colloidal suspension [42]. In order to improve the method, further research will be carried out in this direction.

\section{Conclusions}

Feasibility of an air pressurized system to reduce the size of silver nanoparticles by laser radiation is demonstrated. Crystalline Ag nanoparticles have been obtained by means of LASL and re-irradiation technique using two different nanosecond $\mathrm{Nd}: \mathrm{YVO}_{4}$ lasers working at $532 \mathrm{~nm}$ and $1064 \mathrm{~nm}$ of wavelength, without any chemical reagent or contamination. The particles obtained by LASL exhibit rounded shape. After reirradiation, nanoparticle size is reduced, nevertheless part of the fractured particles coalescence leading to the formation of larger nanoparticles or chains-like nanostructures with a wide range of sizes. All particles show very strong tendency to agglomeration. This is consistent with the metallic nature of the material and the thermal formation mechanisms. 
Despite the fact that both wavelengths reduce the average size of the nanoparticles by means of the re-irradiation process, the number of particles with a diameter smaller than $10 \mathrm{~nm}$ increases considerably if the wavelength used is $1064 \mathrm{~nm}$.

The antibacterial study showed that $s$. aureus is susceptible to the obtained silver nanoparticles, reaching the best inhibitory effects those nanoparticles obtained by laser ablation and re-irradiation with $1064 \mathrm{~nm}$ of wavelength. This result confirms the influence of size, shape and stability in the bactericidal effects of silver nanoparticles.

\section{Acknowledgements}

This work was partially supported by the EU research project CVmar+i (INTERREG V A España-Portugal (POCTEP), by the Government of Spain (MAT2015-71459-C2-P (MINECO/FEDER), PRX17/00157) and by Xunta de Galicia ((ED431B 2016/042, ED481D 2017/010, ED481B 2016/047-0). The technical staff from CACTI (University of Vigo) is gratefully acknowledged. 


\section{References}

[1] J. O'Neil, The Review on Antimicrobial Resistance - Tackling drug resistant infections globally: Final report and recommendations, (2016). Electronic version can be find at https://amr-review.org/ web page.

[2] M.J. Hajipour, K.M. Fromm, A.A. Ashkarran, D.J. de Aberasturi, I.R.de Larramendi, T. Rojo, V. Serpooshan, W.J. Parak, M. Mahmoudi, Antibacterial properties of nanoparticles. Trends in Biotechnology, 30 (2012) 499-511.

[3] I. Sondi, B. Salopek-Sondi, Silver nanoparticles as antimicrobial agent: a case study on E. coli as a model for Gram-negative bacteria. Journal of Colloid and Interface Science 275 (2004) 177-182.

[4] A. Petica, S. Gavriliu, M. Lungu, N. Buruntea, C. Panzaru, Colloidal silver solutions with antimicrobial properties, Mater. Sci. Eng. B Solid-State Mater. Adv. Technol. 152 (2008) 22-27. doi:10.1016/j.mseb.2008.06.021.

[5] S. Chernousova, M. Epple, Silver as antibacterial agent: Ion, nanoparticle, and metal, Angew. Chemie - Int. Ed. 52 (2013) 1636-1653. doi:10.1002/anie.201205923.

[6] S. Pal, Y.K. Tak, J.M. Song, Does the antibacterial activity of silver nanoparticles depend on the shape of the nanoparticle? A study of the gram-negative bacterium Escherichia coli, J. Biol. Chem. 290 (2015) 1712-1720. doi:10.1128/AEM.02218-06.

[7] B. Perito, E. Giorgetti, P. Marsili, M. Muniz-Miranda, Antibacterial activity of silver nanoparticles obtained by pulsed laser ablation in pure water and in chloride solution, Beilstein J. Nanotechnol. 7 (2016) 465-473. doi:10.3762/bjnano.7.40.

[8] M. Ratti, J.J. Naddeo, Y. Tan, J.C. Griepenburg, J. Tomko, C. Trout, S.M. O’Malley, D.M. Bubb, E.A. Klein, Irradiation with visible light enhances the antibacterial toxicity of silver nanoparticles produced by laser ablation, Appl. Phys. A. 122 (2016) 346. doi:10.1007/s00339-016-9935-8. 
[9] Y.G. Yuan, Q.L. Peng, S. Gurunathan, Effects of silver nanoparticles on multiple drug-resistant strains of Staphylococcus aureus and Pseudomonas aeruginosa from mastitis-infected goats: An alternative approach for antimicrobial therapy, Int. J. Mol. Sci. 18 (2017). doi:10.3390/ijms18030569.

[10] C. Marambio-Jones, E.M.V. Hoek, A review of the antibacterial effects of silver nanomaterials and potential implications for human health and the environment, J. Nanoparticle Res. 12 (2010) 1531-1551. doi:10.1007/s11051-010-9900-y.

[11] M. Guzman, J. Dille, S. Godet, Synthesis and antibacterial activity of silver nanoparticles against gram-positive and gram-negative bacteria, Nanomedicine Nanotechnology, Biol. Med. 8 (2012) 37-45. doi:10.1016/j.nano.2011.05.007.

[12] J.W. Rhim, L.F. Wang, Y. Lee, S.I. Hong, Preparation and characterization of bionanocomposite films of agar and silver nanoparticles: Laser ablation method, Carbohydr. Polym. 103 (2014) 456-465. doi:10.1016/j.carbpol.2013.12.075.

[13] A. Panacek, L. Kvítek, R. Prucek, M. Kolár, R. Vecerová, N. Pizúrová, V.K. Sharma, T. Nevecna, R. Zboril. Silver Colloid Nanoparticles: Synthesis, Characterization, and Their Antibacterial Activity, J. Phys. Chem. B (2006) $16248-16253$.

[14] K.H. Tseng, Y.C. Chen, J.J. Shyue, Continuous synthesis of colloidal silver nanoparticles by electrochemical discharge in aqueous solutions, J. Nanopart. Res. 13 (2011) 1865-1872.

[15] M.N. Nadagouda, T.F. Speth, R.S. Varma, Microwave-assisted green synthesis of silver nanostructures, Acc. Chem. Res. 44 (2011) 469-478.

[16] J.L. López-Miranda, M. Vázquez, N. Fletes, R. Esparza, G. Rosas, Biosynthesis of silver nanoparticles using a Tamarix gallica leaf extract and their antibacterial activity, Mater. Lett. 176 (2016) 285-289. 
[17] I. Ghiuță, D. Cristea, C. Croitoru, J. Kost, R. Wenkert, I. Vyrides, A. Anayiotos, D. Munteanu, Characterization and antimicrobial activity of silver nanoparticles, biosynthesized using Bacillus species, Appl. Surf. Sci. 438 (2017) 66-73. doi:10.1016/j.apsusc.2017.09.163.

[18] S. Barcikowski, G. Compagnini, Advanced nanoparticle generation and excitation by lasers in liquids, Phys.Chem. Chem. Phys., 15 (2013) 3022.

[19] S. Barcikowski, S., A. Méndez-Manjón, B. Chichkov, M. Brikas, G. Raciukaitis, Generation of nanoparticle colloids by picosecond and femtosecond laser ablations in liquid flow, Appl. Phys. Lett. 91 (2007), 083113.

[20] B. Xu, R.G. Song, Fabrication of Ag nanoparticles colloids by pulsed laser ablation in liquid, Avanced Materials Research, 123-125 (2010) 675-678.

[21] M.I. Mendivil, B. Krishnan, F.A. Sánchez, S. Martínez, J.A. Aguilar-Martínez, G.A. Castillo, D.I. García-Gutiérrez, S. Shaji, Synthesis of silver nanoparticles and antimony oxide nanocrystals by pulsed laser ablation in liquid media, Appl Phys A 110 (2013) 809-816.

[22] S.V. Rao, G.K. Podagatlapalli, S. Hamad, Ultrafast laser ablation in liquids for nanomaterials and applications, J. Nanosci Nanotechnol. 14 (2014) 1364-1388.

[23] M.A. Valverde-Alva, T. García-Fernández, M. Villagrán-Muniz, C. SánchezAké, R. Castañeda-Guzmán, E. Esparza-Alegría, C.F. Sánchez-Valdés, J.L.S. Llamazares, C.E.M. Herrera, Synthesis of silver nanoparticles by laser ablation in ethanol: A pulsed photoacoustic study, Appl. Surf. Sci. 355 (2015) 341-349.

[24] M. Dell'Aglio, V. Mangini, G. Valenza, O. De Pascale, A. De Stradis, G. Natile, G.,F. Arnesano, A. De Giacomo, Silver and gold nanoparticles produced by pulsed laser ablation in liquid to investigate their interaction with Ubiquitin, Appl. Surf. Sci., 374 (2016) 297-304. 
[25] A. Resano-García, S. Champmartin, Y. Battie, A. Koch, A. En Naciri, A. Ambari, N. Chaoui, Highly-repeatable generation of very small nanoparticles by pulsed-laser ablation in liquids of a high-speed rotating target, Phys.Chem.Chem.Phys., 18 (2016) 32868.

[26] S. Kohsakowski, A. Santagata, M. Dell'Aglio, A. de Giacomo, S. Barcikowski, P. Wagener, B. Gökce, High productive and continuous nanoparticle fabrication by laser ablation of a wire-target in a liquid jet, Appl. Surf. Sci. 403 (2017) 487-499.

[27] M. Boutinguiza, R. Comesaña, F. Lusquiños, A. Riveiro, J. Del Val, J. Pou, Production of silver nanoparticles by laser ablation in open air, Appl. Surf. Sci. 336 (2015) 108-111. doi:10.1016/j.apsusc.2014.09.193.

[28] M. Boutinguiza, M. Fernández-Arias, J. del Val, J. Buxadera-Palomero, D. Rodríguez, F. Lusquiños, F.J. Gil, J. Pou, Synthesis and deposition of silver nanoparticles on cp Ti by laser ablation in open air for antibacterial effect in dental implants, Mater. Lett. 231 (2018) 126-129. doi:10.1016/j.matlet.2018.07.134.

[29] P. Wagener, S. Barcikowski, Laser fragmentation of organic microparticles into colloidal nanoparticles in a free liquid jet, Appl Phys A 101 (2010) 435-439.

[30] R. Salomoni, P. Léo, M.F.A. Rodrigues, Antibacterial Activity of Silver Nanoparticles (AgNPs) in Staphylococcus aureus and Cytotoxicity Effect in Mammalian Cells, Battle Against Microb. Pathog. Basic Sci. Technol. Adv. Educ. Programs. (2015) $851-857$.

[31] D.S. Ondusko, D. Nolt, Staphylococcus aureus, Pediatr. Rev. 39 (2018) 287-298. doi:10.1542/pir.2017-0224.

[32] F. Luo, Y. Guan, W. Ong, Z. Du, G. Ho, F. Li, S. Sun, G. Lim, M. Hong, Enhancement of pulsed laser ablation in environmentally friendly liquid, Opt. Express. 22 (2014) 23875. doi:10.1364/OE.22.023875. 
[33] J. Jeon, S. Yoon, H.W. Choi, J. Kim, D. Farson, The Effect of Laser Pulse Widths on Laser - Ag Nanoparticle Interaction : Femto- to Nanosecond Lasers, Appl. Sci. 8 (2018) 13. doi:10.3390/app8010112.

[34] F. Mafuné, J. Kohno, Y. Takeda, T. Kondow, Hisahiro Sawabe, Formation and Size Control of Silver Nanoparticles by Laser Ablation in Aqueous Solution, J. Phys. Chem. B. 104 (2000) 9111-9117. doi:10.1021/jp001336y.

[35] V. Amendola, M. Meneghetti, Laser ablation synthesis in solution and size manipulation of noble metal nanoparticles, (2009) 3805-3821. doi:10.1039/b900654k.

[36] S. Dadras, M.J. Torkamany, P. Jafarkhani, Analysis and optimization of silver nanoparticles laser synthesis with emission spectroscopy of induced plasma, J. Nanosci. Nanotechnol. 12 (2012) 3115-3122. doi:10.1166/jnn.2012.5830.

[37] S. Bhattacharjee, DLS and zeta potential - What they are and what they are not?, J. Control. Release. 235 (2016) 337-351. doi:10.1016/j.jconrel.2016.06.017.

[38] J.R. Morones, J.L. Elechiguerra, A. Camacho, K. Holt, J.B. Kouri, J.T. Ramírez, M.J. Yacaman, The bactericidal effect of silver nanoparticles, Nanotechnology. 16 (2005) 2346-2353. doi:10.1088/0957-4484/16/10/059.

[39] G. V. Vimbela, S.M. Ngo, C. Fraze, L. Yang, D.A. Stout, Antibacterial properties and toxicity from metallic nanomaterials, Int. J. Nanomedicine. 12 (2017) 3941-3965. doi:10.2147/IJN.S134526.

[40] Z.M. Xiu, Q.B. Zhang, H.L. Puppala, V.L. Colvin, P.J.J. Alvarez, Negligible particle-specific antibacterial activity of silver nanoparticles, Nano Lett. 12 (2012) 42714275. doi:10.1021/nl301934w.

[41] N. Sheikh, A. Akhavan, M.Z. Kassaee, Synthesis of antibacterial silver nanoparticles by $\gamma$-irradiation, Phys. E Low-Dimensional Syst. Nanostructures. 42 (2009) 132-135. doi:10.1016/j.physe.2009.09.013. 
[42] C. Rehbock, V. Merk, L. Gamrad, R. Streubel, S. Barcikowski, Size control of laser-fabricated surfactant-free gold nanoparticles with highly diluted electrolytes and their subsequent bioconjugation. Phys.Chem. Chem. Phys., 2013, 15, 3057. 